Research Article

\title{
Backlog to Surgery due to COVID- 19 and Considerations to Address to Catch-up Safely
}

\author{
Amy Lewis', Benjamin Polatidis' ${ }^{2}$ Evi Charitou ${ }^{3}$, Anna Karapatsia ${ }^{4}, \underline{\text { Anne Ahlmann Nielsen }}^{5}$, \\ Christian Bjerregaard Olesen ${ }^{6}$, Athanasios Apostolos Gargavanis $^{7}$, Sara Halimah $^{8}$, \\ $\underline{\text { Georgios loannis Tsianos }^{9}}$, Doros Polydorou $^{10}, \underline{\text { Katharine Holt }}^{11}, \underline{\text { Marinela Cebic }}^{12}$, \\ Efstathios Vassiliadis ${ }^{13}$
} 1,2,3,4,5,6,9,13Evnia, Copenhagen Business Centre, Hellerup Strandvejen 60, DK-2900, Copenhagen, Denmark.
7,8 World Health Organization, OPT, Emergency Care Technical Officer, Jerusalem, Israel.
10,11, 12Steris, Rutherford House, Stephenson's Way, Derby, DE21 6LY, United Kingdom.
DOI: https://doi.org/10.24321/2394.6539.202010

I $\quad \begin{array}{lllll}\mathbf{N} & \mathbf{F} & \mathbf{O}\end{array}$

Corresponding Author:

Amy Lewis, Copenhagen Business Centre, Hellerup Strandvejen 60, DK-2900, Copenhagen, Denmark.

E-mail Id:

amle@evnia.dk

Orcid Id:

https://orcid.org/0000-0001-8616-2411

How to cite this article:

Lewis A, Polatidis B, Karapatsia A, Charitou E, Nielsen $A A$, Olesen $C B$ et al. Backlog to Surgery due to COVID-19 and Considerations to Address to Catch-up Safely. J Adv Res Med Sci Tech 2020; 7(3): 4-10

Date of Submission: 2020-09-09

Date of Acceptance: 2020-09-23

\section{$\begin{array}{lllllllllllllll}\mathbf{A} & \mathbf{B} & \mathbf{S} & \mathbf{T} & \mathbf{R} & \mathbf{A} & \mathbf{C} & \mathbf{T}\end{array}$}

On March $11^{\text {th }} 2020$, COVID-19 met all criteria to be considered a pandemic. The resulting halt in surgery led to an estimated 28.4 million elective surgery cancellations around the globe. Figures suggest that the backlog to surgery may take years to clear, causing huge detrimental effects to both the economy and the health of patients. Addressing this surgical backlog, whilst ensuring the health and safety of healthcare professionals and patients during surgery, will require a multifaceted approach. Key aspects, such as providing a sufficient and stable supply of Personal Protective Equipment (PPE), adequate and efficient testing of healthcare staff and patients, and availability of post-acute care, need to be secured before surgery can recommence safely in this COVID-19 era.

Keywords: COVID-19, Coronavirus, Surgery, Surgery Backlog, Surgery Cancellation

\section{COVID-19 Backlog to Surgery}

The halt in surgery began when countries around the world became affected by COVID-19. On $30^{\text {th }}$ January 2020, the World Health Organization (WHO) declared COVID-19 as a Health Emergency of International concern. By March $11^{\text {th }}$ 2020 , it met all criteria to be referred to as a pandemic. This review presents the impact that COVID-19 has had on surgery around the globe, and provides suggestions for ways to tackle the resulting backlog to surgery.

\section{Surgery Cancellations}

As a result of insufficient resources to manage a novel virus like COVID-19, essential resources, such as PPE, testing materials, ventilators and intensive care unit (ICU) beds, needed to be diverted, resulting in global surgery cancellations. These cancellations were necessary "in order to ensure sufficient capacity for COVID-19 demand, and also to allow time to evaluate whether usual volumes of surgery could continue safely in the context of COVID-19 
without risk of infection to patients". ${ }^{1}$ However, the resulting effect was a tremendous increase in waiting lists for surgeries worldwide, causing critical cash shortages for many hospitals deprived of a significant amount of their scheduled revenue. ${ }^{2}$

\section{Canada}

Figures in Canada show that most elective surgeries have been cancelled since mid-March, causing major delays in patient care, mounting to an evitable peak demand on the health system. ${ }^{1}$ If cancellations continue for a 12 week period, 394,576 surgeries will be cancelled, including 27,390 cancer procedures. It is expected that this backlog will take 11-months to clear if the number of surgeries performed each week can be increased by 20 percent (compared to pre-pandemic activity)..$^{1}$ In British Columbia, it has been reported that approximately 30,000 surgeries were cancelled between $16^{\text {th }}$ March and $18^{\text {th }}$ May, of which 14,000 were postponed and 16,000 not yet rescheduled. ${ }^{3}$ It is estimated that this 2-month backlog alone will take as long as 2 years to clear. ${ }^{3}$

\section{United Kingdom}

In the United Kingdom (UK), the British Heart Foundation recently estimated that 28,000 heart related procedures (including implanting of pacemakers or stents, widening blocked arteries to the heart, and tests to diagnose heart problems) have been delayed since the outbreak of COVID-19. ${ }^{4}$ Furthermore, with the National Health Service (NHS) surgery wait-lists topping 4 million pre COVID-19, this number is expected to double by the end of June, ${ }^{5}$ putting further pressure on an already fragile and depleting NHS. Such cancellations are reflected in reduced surgical instrument processing volumes early on in the pandemic. Primary data provided by STERIS, a world known provider of procedural products and services, reported that processing volumes in the UK of sterilized surgical trays depleted to $20 \%$ on average at the beginning of April and have only slowly begun to rise since July (see Figure 1). This trend in processing highlights the impact COVID-19 has had on surgery.

\section{United States}

Research in the United States (US) has also begun to generate predictions regarding the time it will take to clear the backlog. Using a stochastic simulation-based analysis, Jain et al. ${ }^{6}$ identified that it will take 7, 12 or 16 months (using an optimistic, ambivalent or pessimistic scenario, respectively) for the US healthcare system to recover enough to allow $90 \%$ of elective orthopaedic procedures to be performed, assuming procedures resume in June 2020. They also identified a "cumulative backlog of $>1$ million surgical cases at 2 years after the end of deferment" in the optimistic scenario. ${ }^{6}$ Additionally, Salenger et al. ${ }^{7}$ estimate that the backlog in adult cardiac surgery programs in 2 healthcare systems in the US will require between 1 and 8 months to clear. The time range depends upon when surgery can resume, as well as the extent of the post-pandemic increased operational capacity. The authors also calculated a decrease in cardiac surgery procedures of nearly $50 \%$ during the pandemic. ${ }^{7}$

\section{Gaza}

While a backlog of elective surgical procedures is rather new in Western World countries, it is not uncommon for regions with limited health resources. In Gaza, a place under blockade since $2007,{ }^{8}$ postponement of elective surgical operations is commonplace, impacting patient wellbeing and increasing the risk of complications. In 2018, according to the Ministry of Health, more than 8000 elective surgical operations were postponed due to an insufficient health workforce, depleting medical equipment and supplies, and limited infrastructure capacity. ${ }^{9}$ Before these needs could be addressed, COVID-19 imposed additional burdens within all four pillars of trauma care (pre-hospital care, acute care, specialised care, rehabilitation care). ${ }^{10}$ Areas affected include the postponement of mass-casualty management training and the deployment of international emergency medical teams to support trauma surgery. Further decreases in selective surgical activities have also occurred, with a direct impact on the continuous, decreasing number of patients undergoing limb reconstruction surgery. ${ }^{10}$ Selective surgical activities at the Al Awda Hospital and the Al Amal

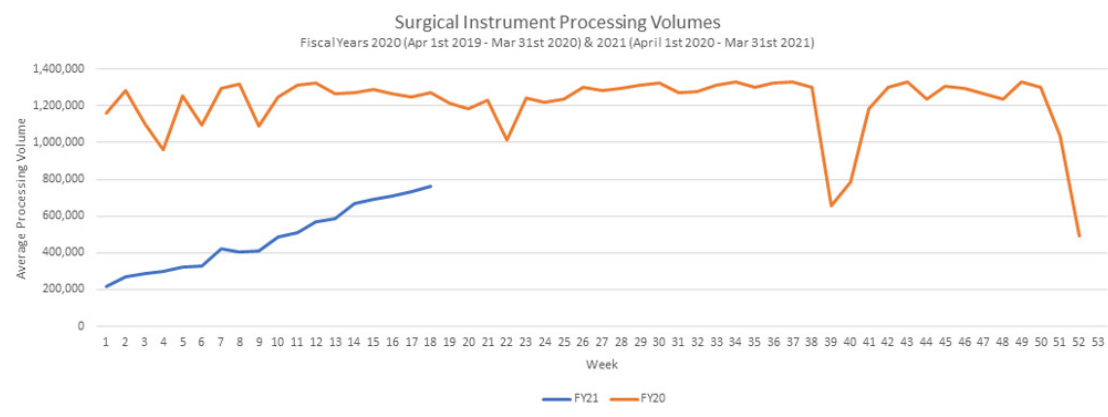

Figure I.STERIS surgical instrument processing volumes in the UK for fiscal years 20 and 2I. Data is taken from SynergyTrak ${ }^{\circledR}$, the operations software used by STERIS 
Palestinian Red Crescent Society Hospital in Gaza have been decreased by $50 \%$ and $100 \%$, respectively. Although specialized training on PPE use has been delivered to health professionals in the Al Awda hospital, limited access to PPE is increasing the risk of infection for paramedics and health specialists, all along the Gaza Strip ${ }^{10}$

\section{The Cost of Surgery Cancellations}

The backlog to surgery is a worldwide issue that has already resulted in high financial costs. In England alone, the estimated cost of cancelled operations has been predicted to be between $£ 5,045,354,856$ and $£ 5,231,861,474$ by February $28^{\text {th }} 2021 .^{11}$ Furthermore, delayed surgeries have likely led to the deterioration of patients with clinical health issues, which will result in more costly and lengthy surgeries $^{5,12}$ (see Figure 2 for an example of estimated costs). It has been calculated that delay in cancer patients' treatment could result in dramatic reduction in long-term survival, with over $30 \%$ survival reduction for those with advanced stage cancers. ${ }^{13}$ Treatment delay of low-grade tumours could result in more advanced cancer, impacting both patient survival and healthcare costs. ${ }^{13}$ Additionally, delays to metabolic and bariatric surgeries have potentially deleterious long-term effects with regards to morbidity and mortality, if conditions associated with obesity or diabetes are left untreated. ${ }^{14}$

\section{Clearing the Backlog to Surgery}

To address this surgical backlog, in addition to ensuring the safety of patients and staff during surgery in the COVID-19 era, multiple approaches need to be considered (see Figure 3).

\section{International Research and Collaboration}

In the face of this unprecedented threat, research and international collaboration is crucial to reduce the impact of COVID-19 on our health systems. ${ }^{1}$ As such, surgeons from around the world have begun to unite to protect patients through an international cohort study named CovidSurg. ${ }^{15,16}$ The GlobalSurg Collaborative established CovidSurg on $28^{\text {th }}$ March 2020, with the aim of assessing outcomes after surgery in COVID-19 patients by capturing real-world data from around the globe. ${ }^{15,16}$ The ethos of CovidSurg is inclusive and collaborative - an international study which is open to all collaborators from anywhere in the world. ${ }^{16}$ Within just a few days, CovidSurg had received 1,355 registrations from more than 96 countries; this number continues to rise. ${ }^{17}$

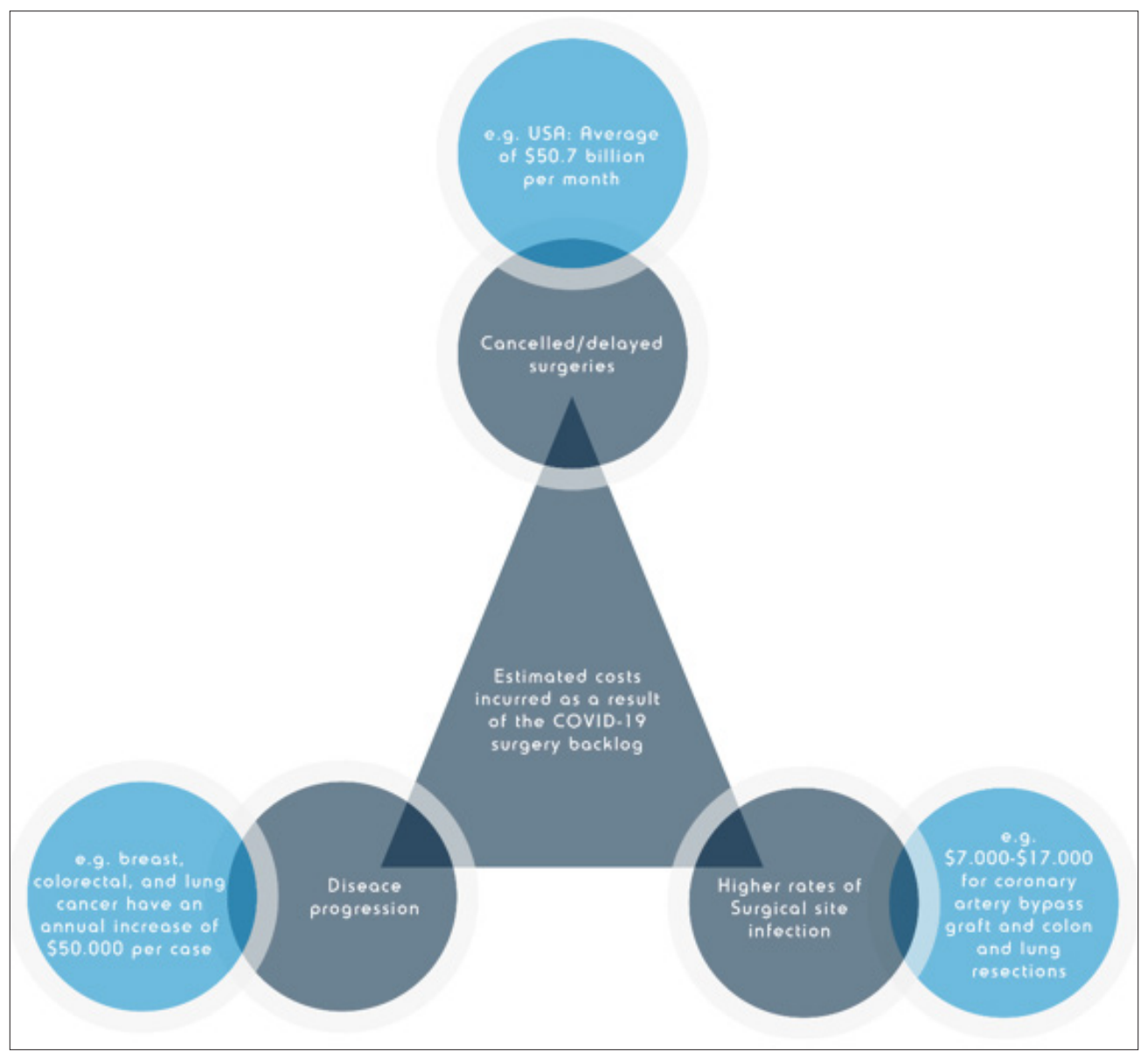

Figure 2.Estimated costs as a result of the surgery backlog. Information sourced from ${ }^{12,22}$ 


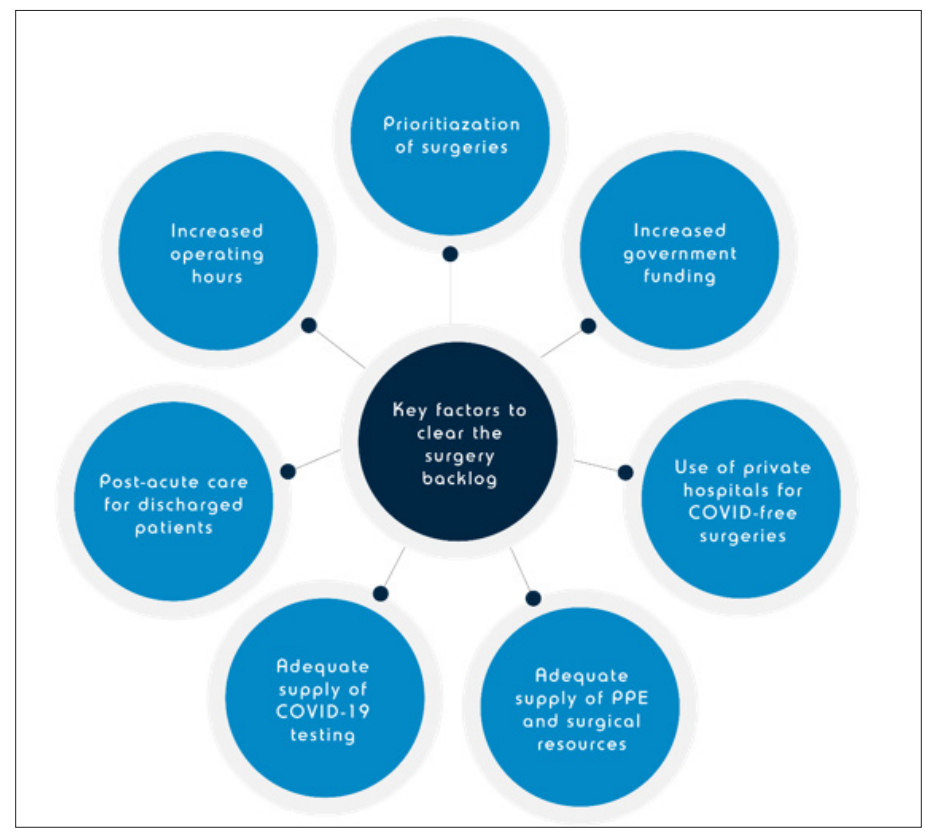

Figure 3.Key factors to clear the surgery backlog. Information sourced from $1,7,23,25,26,24$

The efforts of CovidSurg have, to date, resulted in three published articles: mortality and pulmonary complications in patients undergoing surgery with SARS-CoV- $2,{ }^{18}$ global guidance for surgical care during the COVID-19 pandemic, ${ }^{19}$ and elective surgery cancellations due to COVID-19. ${ }^{20}$ The latter is a global expert study that was conducted to elicit projections of elective surgeries that would be cancelled or postponed during a 12-week period of peak disruption. ${ }^{20}$ The study was first launched on $15^{\text {th }}$ April 2020 and published in the British Journal of Surgery on $12^{\text {th }}$ May $2020 .{ }^{20}$ It estimated that 2.4 million surgeries would be cancelled globally per week, the equivalent of 28.4 million (or $72.3 \%$ of procedures) over a 12 -week period (see Figure 4). Benign procedures were predicted to be the most frequently cancelled (estimated at 25 million cancellations worldwide; $90.2 \%$ of global cancellations), with cancer surgery cancellations estimated at 2.3 million (8.2\% of global cancellations).

The 12-week estimate was based on the experience of China's Hubei Province, where the virus originated. ${ }^{20}$ Janet Martin, who is part of the CovidSurg research, explained that " $(t)$ his has never happened before on a global basis in an era where we have the volume of surgery we have today. Surgery is a critically important part of health care globally. This study was essential to define the global impact of surgical cancellations". ${ }^{1}$ To date, there are no other studies of the kind, and the article has been cited in the popular press around the world. ${ }^{1,21,3}$

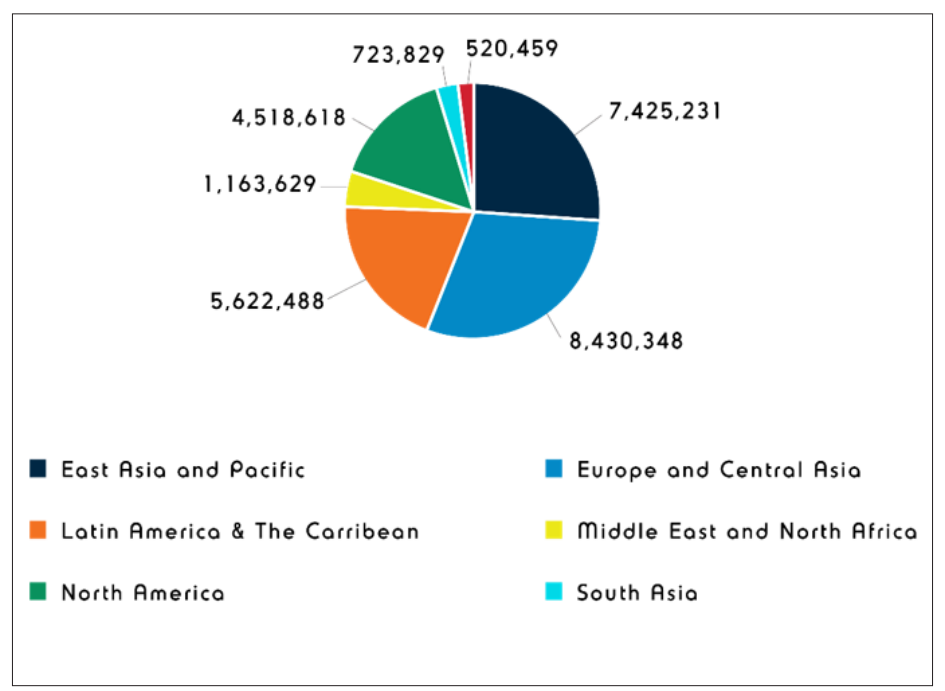

Figure 4.Projected worldwide surgery cancellations over a I2-week period of peak disruption. Adapted from ${ }^{20}$ 


\section{Increasing Operating Hours}

Increasing operating hours may be an option to help alleviate the surgery backlog. In Canada, daily operating hours will be increased, including scheduling procedures on evenings and weekends. Additionally, new Operating Rooms (ORs) and unused ORs will be (re) opened. ${ }^{1}$ However, this is unlikely to be a sustainable solution, due to the need for healthcare professionals to increase their hours and the subsequent cost of paying them to do so. ${ }^{1}$ Although surgical overtime may be supported by governmental funding in Canada, ${ }^{23}$ the physical demands of the extra hours for staff must be considered; with staff in the UK already at "absolute exhaustion", ${ }^{24}$ this is not a viable option for all counties.

\section{Use of Private Hospitals}

Another strategy is to contract additional private clinics and hospitals. Canada plans to do this as long as the private clinics follow the Canada Health Act and do not charge patients extra. ${ }^{1}$ The use of private hospitals as COVID-free surgery points for vulnerable patients' groups has already been implemented in UK; however, reports suggest that these hospitals are not being used, with very few patients being referred to private hospitals. ${ }^{25}$

\section{Single-entry Model}

For patients in need of cardiac surgery, it is proposed that "maximizing efficiency through early telemetry discharge, decreasing intensive care length of stay, level loading elective cases in the OR, temporarily altering staffing models, and staggering cases can help increase capacity". ${ }^{27}$ For elective surgical procedures, an effective strategy to reduce the backlog may be to switch to a single-entry model. ${ }^{28}$ This is a system that, once a patient is referred to the hospital for a procedure, allocates an available surgeon to a patient, instead of a surgeon that is selected/ preferred by the patient and may be otherwise unavailable. A singleentry model could also include "pooled/ common waiting lists (consolidation of multiple waiting lists); centralised intake (a single point-of-entry through which referrals are received and service provision arranged) and triage (through which referrals are assessed for appropriateness and/or urgency)". ${ }^{28}$ The single-entry model has been verified (via systematic reviews) to be an effective strategy to decrease patient waiting times and waiting lists, and received overall positive patient satisfaction reports. ${ }^{28}$

\section{Stable Supply of PPE and COVID- 19 Testing}

A major issue that will need to be addressed is the availability, or lack thereof, of PPE and COVID-19 testing for healthcare professionals. ${ }^{24}$ Additionally, an adequate supply of COVID-19 testing for patients scheduled for surgery will be crucial. ${ }^{23}$ Chris Hopkins, Chief Executive of the NHS Providers, said that shortages of full-length surgical gowns may result in last minute cancellations of surgeries in the UK. Additionally, test results are taking up to 13 days to be received, meaning that asymptotic staff are at risk of spreading the virus. ${ }^{24} \mathrm{~A}$ study at Addenbrooke's Hospital in Cambridge (UK) has revealed this in action, with findings that $3 \%$ of more than 1,000 asymptotic healthcare staff tested positive for COVID-19. ${ }^{29}$ Until staff can be tested regularly and results received promptly, along with addressing the lack of PPE, operating teams cannot safely perform surgery. ${ }^{24}$ This concern has been reiterated by Dr Rob Harwood, chair of the British Medical Association's consultants committee, who said, "While the PPE situation is improving, we need a guarantee that there is adequate equipment to protect all staff before we recommence services. This includes equipment for those delivering non-Covid care in order to reduce the risk of transmission through health settings. Staff must have confidence that they will be properly protected from infection now and into the future". ${ }^{24}$

Similar concerns in Canada have led the Ontario government to make a list of measures that must be in place before surgery can recommence, including: a stable supply of PPE and medicines, an adequate capacity of inpatient and ICU beds, and the availability of post-acute care outside the hospital to support discharged patients. ${ }^{30}$ Other similar plans are in place in the UK $^{31}$ and Ohio. ${ }^{32}$ Likewise, a recently published joint statement from the American College of Surgeons and others provides guidance on when to safely reopen operating room capacity and includes recommendations for testing, PPE, and case prioritization. ${ }^{33}$

It is also important to note that even when PPE supplies increase, their use must be rational. ${ }^{34} \mathrm{~A}$ recent publication has developed a decision tree algorithm for PPE use in order to protect operating-team staff whilst conserving PPE supplies, with the authors stating that "crisis-era resource consciousness should become part of surgery's new normal." ${ }^{35}$ The availability of resources in general, such as surgical instruments, anaesthesia supplies, ICU capacity and other surgical theatre equipment, will also be a limiting factor to the full recovery, due to the interruptions in manufacturing and/or transport processes during the pandemic. ${ }^{6}$ It is expected that once operations resume, there will be great competition for hospital supplies, human resources and operating rooms between the different surgical and medical (sub)specialties. ${ }^{7}$

\section{Prioritization of Surgeries}

Given the extent of the backlog and limitation on resources, prioritization of surgeries will be necessary. A scoring system for Medically Necessary Time-Sensitive (MeNTS) procedures has recently been published to help with this prioritization, incorporating resource limitations, patient risk and ethical necessity. ${ }^{26}$ In addition, there are guidelines in numerous countries regarding elective surgery for patients that have 
tested positive for COVID-19 (e.g., Spain ${ }^{36}$ and Italy ${ }^{37}$ ), with many advising that surgery should be postponed until the patient has been asymptomatic for 72 hours and has received a least 2 negative COVID-19 tests, 24 hours apart. ${ }^{38}$ Testing for COVID-19 pre-surgery is also recommended, as an asymptotic patient undergoing surgery is at risk of increased complications and mortality. ${ }^{39}$

\section{Concluding Remarks}

Clearly, tackling the backlog to surgery caused by COVID-19 will not be an easy nor straightforward task, and one which will be even more difficult in settings with limited resources. A series of key parameters will need to be addressed, including: increasing governmental funding to cover increased operating hours, contracting private clinics to provide COVID-free surgery points, adequate supply of COVID-19 testing for patients and staff, adequate PPE for healthcare professionals delivering COVID and non-COVID care, post-acute care to reduce the number of patients in the hospital, and prioritization of surgery cases. Moreover, close monitoring and surveillance on outcomes and emphasis on safety of both patients and healthcare professionals will be necessary and crucial for surgery in the COVID-19 era. Awareness should also be made regarding the impact of COVID-19 on low and medium-income countries. In order to limit the death toll and counteract the anticipated negative impact on their economic growth prospects, global surgery efforts need to scale-up procedures in these populations. ${ }^{40}$ COVID-19 threatens to demise this upscale, due to the diversion of health-care resources. ${ }^{40}$ Research suggests that the impact of the pandemic is expected to cause great stress on the workforce, service delivery and infrastructures of low and medium-income populations and that "(i)f robust progress towards global surgery goals for 2030 is to continue, the stress points identified should be reinforced." 40

\section{Acknowledgement}

The authors wish to thank STERIS for providing primary data on surgical instrument processing volumes.

\section{Funding}

The Authors declare that there is no funding.

\section{Conflicts of Interest: None}

\section{References}

1. Mackay C: Study: 28 million surgeries shelved by COVID-19. Western News May 14th, 2020

2. Hook L, Kuchler H. How Coronavirus broke America's healthcare system. The Financial Times 30th April, 2020. https://www.ft.com/content/3bbb4f7c-890e11ea-a01c-a28a3e3fbd33.

3. Coyne T. B.C. estimates 2-year backlog in surgeries due to COVID-19. Vancouver Island News 2020. https:// vancouverisland.ctvnews.ca/b-c-estimates-2-yearbacklog-in-surgeries-due-to-covid-19-1.4928346

4. BBC: 'Tens of thousands' of heart procedures delayed by pandemic 2020. https://www.bbc.com/news/ health-52923771.

5. Independent: NHS on life support: Up to one in six will be on waiting lists as health service turns to private hospitals. Independent 2020. https://www. independent.co.uk/news/health/coronavirus-nhswaiting-times-surgery-privatisation-a9550831.html.

6. Jain A, Jain P, Aggarwal S. SARS-CoV-2 Impact on Elective Orthopaedic Surgery: Implications for PostPandemic Recovery. The Journal of Bone and Joint surgery American 2020.

7. Salenger R, Etchill EW, Ad N, Matthew T, Alejo D, Whitman $\mathrm{G}$ et al. The surge after the surge: cardiac surgery post-COVID-19. The Annals of thoracic surgery 2020.

8. Affairs UNOFTCOH: Gaza blockade. Accessed August 8th, 2020.

9. Organization WH: Emergency Trauma Response to the Gaza Mass Demonstarions 2018-2019. Accessed August 8th, 2020.

10. Gaza Trauma Working Group: Delivering trauma care services in time of COVID-19 May 2020. http://www. healthclusteropt.org/news-and-stories/194.

11. Fowler AJ, Dobbs TD, Wan YI, Laloo R, Hui S, Nepogodiev $D$ et al. Resource requirements for reintroducing elective surgery in England during the COVID-19 pandemic: a modelling study. medRxiv 2020.

12. Fu SJ, George EL, Maggio PM, Hawn M \& Nazerali R. The Consequences of Delaying Elective Surgery: Surgical Perspective. Annals of Surgery 2020.

13. Sud A, Jones M, Broggio J, Loveday C, Torr B, Garrett A et al. Collateral damage: the impact on outcomes from cancer surgery of the COVID-19 pandemic. Annals of Oncology 2020.

14. Rubino F, Cohen RV, Mingrone G, Le Roux CW, Mechanick JI, Arterburn DE et al. Bariatric and metabolic surgery during and after the COVID-19 pandemic: DSS recommendations for management of surgical candidates and postoperative patients and prioritisation of access to surgery. The Lancet Diabetes \& Endocrinology 2020.

15. CovidSurg: Outcomes of surgery in COVID-19 infection: international cohort study (CovidSurg) 2020. https:// clinicaltrials.gov/ct2/show/NCT04323644.

16. NIHR Global Health Research Unit on Global Surgery: About CovidSurg Cohort Study 2020. https://globalsurg. org/covidsurgcohortstudy/

17. Bowel Disease Research Foundation: COVIDSURG collaborative launched to tackle pandemic 2020. https://bdrf.org.uk/news/medicalscientific-news/ 
covidsurg-collaborative-launched-to-tackle-pandemic/.

18. Nepogodiev D, Glasbey JC, Li E, Omar OM, Simoes JF, Abbott TE et al. Mortality and pulmonary complications in patients undergoing surgery with perioperative SARS-CoV-2 infection: an international cohort study. The Lancet 2020.

19. Collaborative C: Global guidance for surgical care during the COVID-19 pandemic. The British journal of surgery 2020.

20. Collaborative C, Nepogodiev D \& Bhangu A: Elective surgery cancellations due to the COVID-19 pandemic: global predictive modelling to inform surgical recovery plans. British Journal of Surgery 2020.

21. Vanguard News Nigeria: Coronavirus could cause 28 million cancelled surgeries globally - Study 2020 https://www.vanguardngr.com/2020/05/coronaviruscould-cause-28-million-cancelled-surgeries-globally\%E2\%80\%95study/.

22. American Hospital Assoication: Hospitals and Health Systems Face Unprecedented Financial Pressures Due to COVID-19 2020.

23. Ireland N: How hospitals will tackle the backlog of nearly 100,000 surgeries delayed by the pandemic. CBC News 2020. https://www.cbc.ca/news/health/ covid-19-surgery-backlog-canada-1.5543530.

24. The Guardian: Huge backlog in operations building due to lack of PPE and staff testing 2020.

25. Boyd C: Private hospitals commandeered for Britain's Covid-19 crisis at a cost of $£ 150$ million are STILL empty - despite huge backlog of NHS patients needing routine surgery and vital cancer treatment. Mail Online 2020. https://www.dailymail.co.uk/news/article-8387187/ Private-hospitals-commandeered-Britains-Covid-19crisis-empty.html.

26. Prachand VN, Milner R, Angelos P, Posner MC, Fung $\mathrm{JJ}$, Agrawal $\mathrm{N}$ et al. Medically-necessary, time-sensitive procedures: a scoring system to ethically and efficiently manage resource scarcity and provider risk during the COVID-19 pandemic. Journal of the American College of Surgeons 2020.

27. Kester KM, Lindsay M, Granger B. Development and evaluation of a prospective staffing model to improve retention. Journal of Nursing Management 2020; 28, 425-432.

28. Damani Z, Conner-Spady B, Nash T, Stelfox HT, Noseworthy TW, Marshall DA. What is the influence of single-entry models on access to elective surgical procedures? A systematic review. BMJ open 2017; 7: e012225.

29. Rivett L, Sridhar S, Sparkes D, Routledge M, Jones NK, Forrest $S$ et al. Screening of healthcare workers for SARS-CoV-2 highlights the role of asymptomatic carriage in COVID-19 transmission. Elife 2020; 9: e58728.
30. Davidson. S \& D'mello C: Ontario unveils plan to resume elective surgeries and procedures. CTV News 2020. https://toronto.ctvnews.ca/ontario-unveils-plan-toresume-elective-surgeries-and-procedures-1.4928705

31. NHS: operating framework for urgent and planned services within hospitals 2020. https://www. england.nhs.uk/coronavirus/wp-content/uploads/ sites/52/2020/05/Operating-framework-for-urgentand-planned-services-within-hospitals.pdf.

32. Poulose BK, Phieffer LS, Mayerson J, Like D, Forrest LA, Rahmanian A et al. Responsible Return to Essential and Non-Essential Surgery During the COVID-19 Pandemic. Journal of Gastrointestinal Surgery 2020: 1.

33. American College of Surgeons ASOA, Association of Perioperative Registered Nurses \& Association TaH. Joint Statement: Roadmap for Resuming Elective Surgery after COVID-19 Pandemic. American Society of Anesthesiologists 2020.

34. World Health O. Rational use of personal protective equipment for coronavirus disease ("COVID-19):: interim guidance, 27 February 2020. Geneva: World Health Organization 2020.

35. Argenziano M, Fischkoff K, Smith CR. Surgery scheduling in a crisis. New England Journal of Medicine 2020.

36. Balibrea JM, Badia JM, Pérez IR, Antona EM, Peña EÁ et al. Surgical management of patients with COVID-19 infection. recommendations of the Spanish association of surgeons. Cirugía Española (English Edition) 2020.

37. Coccolini F, Perrone G, Chiarugi M, Di Marzo F, Ansaloni L et al. Surgery in COVID-19 patients: operational directives. World Journal of Emergency Surgery 2020; 15: 1-7.

38. Sharma D, Agrawal V, Agarwal P. Roadmap for Restarting Elective Surgery During/After COVID-19 Pandemic. Indian Journal of Surgery 2020: 1-5.

39. Nahshon C, Bitterman A, Haddad R, Hazzan D, Lavie O. Hazardous postoperative outcomes of unexpected covid-19 infected patients: a call for global consideration of sampling all asymptomatic patients before surgical treatment. World Journal of Surgery 2020: 1.

40. Mazingi D, Navarro S, Bobel MC, Dube A, Mbanje C \& Lavy C: Exploring the Impact of COVID-19 on Progress Towards Achieving Global Surgery Goals. World Journal of Surgery 2004: 1. 